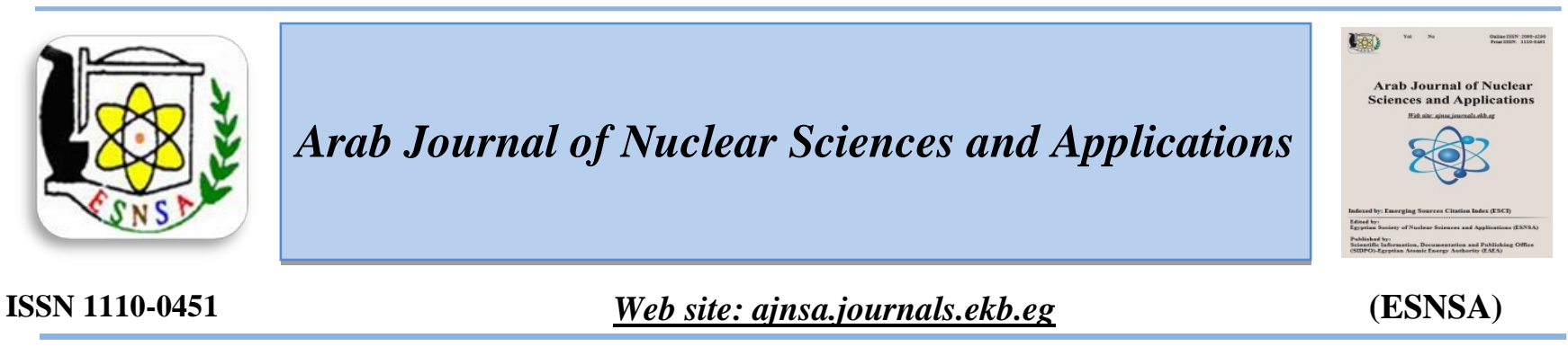

\title{
Production of Polyhydroxybutyrate (PHB) from Streptomyces incanus and the Effect of Gamma Irradiation
}

\author{
A.E. Doaa ${ }^{2}$, M.A. Swelim ${ }^{1}$, H.M. Swailam ${ }^{2}$ and A.R. Samar ${ }^{1}$ \\ ${ }^{1}$ Botany and Microbiology Department, Faculty of Science, Benha University, Egypt \\ ${ }^{2}$ Radiation Microbiology Dept., National Center for Radiation Research and Technology (NCRRT), AEA, \\ P.O.Box 29 Nasr City, Cairo, Egypt
}

\begin{abstract}
Received $7^{\text {th }}$ Sept. 2019 Polyhydroxybutyrate (PHB) is a biosynthetic and biodegradable plastic which attracts attention du to Accepted $5^{\text {th }}$ March its properties. Out of nine Actinomycetes isolates obtained from different soil samples, the PHB accumulating isolate was identified by molecular studies and was identified as Streptomyces incanus BK128. Different agricultural waste (wheat bran, rice bran, rice straw and molasses) were employed for polyhydroxybutyrate (PHB) production as the main carbon source. Some supplements such as (0.4 g) ammonium sulphate were used to optimize the PHB production medium. The maximum PHB production was achieved with wheat bran $(1.9 \mathrm{~g} / \mathrm{l})$. Different parameters including; the initial $\mathrm{pH}$ of the medium, ammonium chloride concentration and inoculum size; were carried out under shaking flask conditions $(150 \mathrm{rpm})$ at $30^{\circ} \mathrm{C}$ for 5 days of incubation to enhance the PHB production. The maximum PHB production $\left(2.82 \mathrm{gl}^{-1}\right)$ was achieved at $\mathrm{pH} 6.5,(2 \%) \mathrm{NH}_{4} \mathrm{CL}$ and $5 \mathrm{ml}$ inoculum size, respectively. The effect of gamma irradiation on the activity of Streptomyces incanus BK 128 towards PHB production showed that, at dose level $1.5 \mathrm{kGy}$, the maximum PHB production obtained was $3.39 \mathrm{gl}^{-1}$.
\end{abstract}

Keywords: Biopolymer /PHB/ Streptomyces incanus / Wheat bran/ rice bran

\section{Introduction}

Amongst the various existing biodegradable plastic materials, PHB (polyhydroxybutyrate) has achieved foremost consideration due to their structural variety and close correlation to plastics. The fact that PHB can be produced from renewable resources, and their absolute biodegradability quality, makes them the most hopeful product in the near future. PHB has been produced in different scales via fermentation processes as eco- friendly and sustainable plastics [1].
Environment contains abundance of lignocellulosic waste materials and agricultural residues like rice straw, wheat straw, rice bran etc., which could be used for economic PHB production. The major obstruction for the exploitation of lignocellulose is the high stability of this material. Large amounts of lignocellulosic biomass (LB) generated from several agricultural and forest activities may provide inexpensive renewable resources [2]. Molasses is a carbon source for PHB production, and the presence of growth factors especially organic acids, vitamins and minerals in molasses may enhance PHB yield [3].

Corresponding author: newfuture84@hotmail.com

DOI: 10.21608/ajnsa.2020.16698.1266

(C) Scientific Information, Documentation and Publishing Office (SIDPO)-EAEA 
The importance of strain improvement could be achieved either through hybridization and selection within natural population or through population of induced mutant. However, induction of mutation followed by selection has proved to be the most efficient programs for the isolation of highly productive cultures [4]. Bibers and Kalnis, [5] proved that gamma irradiation of mutant of Streptomyces sp. at a dose level of $(0.25-1.5)$ KGy significantly enhanced PHB production.

Therefore, the present study was carried out to produce PHB from a cheap carbon source (waste product agriculture) such as wheat bran, rice straw, molasses and rice bran, and to determine the influence of nutritional conditions as well as the effect of gamma irradiation on the PHB accumulation by the most active organism.

\section{Materials and Methods}

Isolation and selection of Actinomycetes:

Seven Rhizosphere soil samples (lettuce, garlic, onion, zucchini, Eggplant, wheat and alfalfa) were used to isolate Actinomycetes which have the ability to produce PHB using starch nitrate agar medium. For maintenance, the isolates were inoculated into nutrient agar slants and incubated at $30^{\circ} \mathrm{C}$ for 5 days before storage in a refrigerator at $4^{\circ} \mathrm{C}$ for further examination.

Estimation of PHB Production by Staining:

The estimation of PHB was carried out by staining the whole bacterial colonies grown on petri-dishes using Sudan Black B and its modification [7]. Then, the most producer isolate was genetically identified.

\section{Identification of the most active organism}

To characterize the genus of Actinomycete selected as PHB producer, gene-based 16SrRNA identification was used. Molecular identification of the Streptomyces isolate with the highest production of PHB was made. Pure mature culture of the organism for further molecular taxonomic identification using 16S rRNA and primers of the selected isolate according to the National Center for Biotechnology Information (NCBI). This test was conducted in Sigma Laboratory, Cairo, Egypt according to method described by Kaiser et al.[6]. DNA extraction was made according to the Protocol of Gene Jet genomic DNA purification Kit (Thermo K0721). The16S rRNA gene sequence analysis was carried out using NCBI-
BLAST (the National Center for Biotechnology Information) program.

\section{Cultural Conditions:}

For the fermentation in a $250 \mathrm{ml}$ flask culture technique, the standard inoculum of seed culture was added into $100 \mathrm{ml}$ starch nitrate broth medium and incubated at an agitation speed of $150 \mathrm{rpm}$ at $30^{\circ} \mathrm{C}$ for $48 \mathrm{~h}$ in a rotary incubator shaker to initiate the growth [8].

\section{Determination of $\mathrm{PHB}$ production:}

PHB polymer was extracted following the technique previously described by Law et al. [12] and the amount produced was calculated from the standard curve prepared by using commercial poly- $\beta$-hydroxybutyrate (Sigma-Aldrich) [13]. The PHB content was defined as the ratio of PHB concentration to cell concentration given in as a percentage.

\section{PHB Production Parameters:}

Batch fermentations were carried out in $250 \mathrm{ml}$ Erlenmeyer flasks containing $100 \mathrm{ml}$ of culture medium. The flasks were inoculated by the tested organism and maintained at $30^{\circ} \mathrm{C}$ and $150 \mathrm{rpm}$ for 5 days. A set of experiments were performed at different $\mathrm{pH}$ values (5.5-7.5), inoculum size (2-7 $\mathrm{ml})$ and NH4CL concentration (1-4\%) to investigate the effect of these parameters on the PHB production and growth.

Effect of different substrates on PHB production:

A concentrated rice straw solution was used. The solution was prepared according to the technique reported by Sindhu et al. [9]. For pretreatment, the distracted wheat bran (WB) and rice bran were mixed with $1 \% \mathrm{NaOH}$ as reported in a previous study [10]. Molasses were used at $20 \mathrm{~g} / \mathrm{l}$ as a carbon source with or without the addition of 0.8 g/l ammonium sulphate as nitrogen sources [11], and the yield of PHB as well as the growth of Streptomyces incanus BK128 were estimated after 5 days of incubation.

\section{Effect of Gamma Irradiation on production of} PHB:

The effect of different doses of gamma irradiation (0.25-3.5 kGy) on the growth and PHB production were investigated. Irradiation was carried out at the National Center for Radiation Research and 
Technology (NCRRT), using 60Co gamma irradiation source of Russian facility with a dose rate $1.812 \mathrm{kGy} / \mathrm{min}$ at the time of the experiment.

\section{Results and Discussion}

From the obtained 48 isolates which were tested for their ability to produce $\mathrm{PHB}$, nine isolates show the ability to produce PHB. The most active one was genetically identified based on 16SrRNA and showed $100 \%$ of similarity to the genus Streptomyces incanus BK128.

Effect of $p H$ on growth and PHB produced by Streptomyces incanus BK128.

Optimization of batch culture conditions in order to enhance PHB accumulation by Streptomyces incanus BK128 has been studied. The data in Fig.1 revealed that Streptomyces incanus BK128 was able to grow to different degrees in all $\mathrm{pH}$ values from acidic to alkaline starch nitrate medium. The maximum growth and PHB accumulation was achieved at initial $\mathrm{pH} 6.5$ which recorded 4.47and $2.23 \mathrm{gl}^{-1}$ respectively. PHB yield at extreme acidic $\mathrm{pH}$ and extreme alkaline $\mathrm{pH}$ was dramatically decreased. Change in medium $\mathrm{pH}$ may influence the growth of the bacterium, and would influence the PHB production. Typically, a metabolic process may be extremely prone to even minor changes in $\mathrm{pH}$ and therefore, proper control of $\mathrm{pH}$ is critical for PHB production. Hence, PHB production is sensitive to the $\mathrm{pH}$ of cultivation. The most favorable $\mathrm{pH}$ for bacterial growth is neutral $\mathrm{pH}$ (6.5-7.0), and hence contributes to higher PHB production [14].

Effect of inoculum size on growth and PHB produced by Streptomyces incanus BK128.

The effect of the inoculum size on the growth and PHB production was studied. The starch nitrate media were inoculated with different inoculum size $(2-7 \mathrm{ml})$, incubated at $150 \mathrm{rpm}$ and $30^{\circ} \mathrm{C}$ for 5 days. The data represented in Fig.2 illustrated that the cell growth increased from 2.57 to $4.25 \mathrm{~g} / \mathrm{l}$ by increasing the inoculum size from 1 to $5 \mathrm{ml}$. Further increase in inoculum size lead to a slight decrease in cell concentration. On the other hand, the PHB production increased gradually from ( 0.98 to $2.43 \mathrm{~g} / \mathrm{l}$ ) by increasing inoculum size from 1 to $5 \mathrm{ml}$, respectively. Then PHB concentration began to decrease slightly by further increase of inoculum size reaching to $1.85 \mathrm{~g} / \mathrm{l}$ by adding $7 \mathrm{ml}$ inoculum.

Effect of $\mathrm{NH}_{4} \mathrm{CL}$ concentrations on growth and PHB produced by Streptomyces incanus BK128.

The effect of different concentrations of $\mathrm{NH}_{4} \mathrm{CL}$ (1-4\%) on both growth and PHB accumulation was also studied. The data in Fig. 3 revealed that the growth decreased with increasing $\mathrm{NH}_{4} \mathrm{CL}$ concentration from (3-4\%). The maximum growth and PHB production $\left(4.84 \mathrm{gl}^{-1}, 2.82\right)$, respectively was obtained by adding $1.25 \% \mathrm{NH}_{4} \mathrm{CL}$ to the tested medium, and then decreased with further increase in $\mathrm{NH}_{4} \mathrm{CL}$ concentration.

Low nitrogen concentration is good for PHB production. Under normal conditions, bacteria synthesize their cell materials like proteins and can grow well. But in nutrient limiting conditions bacteria may shift their protein synthesis to PHB synthesis for survival. Therefore, nutrient control and environmental stress are required to prompt PHB production. PHB production needs a high carbon source in addition to a nitrogen limitation [15]. Similarly, Thakur et al.[16] worked on the production of PHB and obtained the highest PHB yield in ammonium chloride followed by ammonium sulphate.

Effect of different substrates on growth and $\mathrm{PHB}$ produced by Streptomyces incanus BK128.

Waste materials are frequently important for economic and environmental reasons. The biotechnological utilization of these wastes will eliminate a source of pollution and the products will be obtained cheaply at the same time. For this reason molasses, wheat bran and rice bran were used in the present study as the main carbon source in the production medium. The data given in Fig.(4) demonstrated that the maximum growth was obtained by wheat bran medium $(3.9 \mathrm{~g} / \mathrm{l})$ followed by rice bran $(2.22 \mathrm{~g} / \mathrm{l})$. While, the maximum PHB production was obtained by wheat bran medium (1.9 g/l) followed by rice bran medium with PHB production $(0.95 \mathrm{~g} / \mathrm{l})$. On the other hand, molasses medium recorded unexpectedly the lowest growth and PHB synthesis ( 0.15 and $0.03 \mathrm{~g} / \mathrm{l}$ respectively).

The obtained results indicated that, the maximum PHB production was obtained by wheat bran medium with high PHB content. PHB production had been reported from several bacteria by using waste materials or agricultural residues as 
inexpensive carbon sources [17]. Furthermore, readily available low-price carbon sources may have high amounts of nutrients, such as amino acids and peptides, which contributes to improved cell growth and metabolite biosynthesis and pave the way for resourceful and cost-effective production of PHB. PHB being the reserved food polymer produced during time of starvation, is degraded to provide carbon and energy when external carbon source is exhausted [18].

Various waste materials have also been considered as potential carbon sources for PHB production. Low cost agro-industrial residues like, wheat bran, rice bran have been investigated for PHB production by various researchers [3-10].Molasses, which is a common industrial by-product from sugar industries, is rich in nutrients, minerals and at the same time it is cheap, available in plentiful amounts and could be exploited for production of variety of industrial products [19].

Effect of gamma irradiation on the activity of Streptomyces incanus BK128 towards PHB production.

The effect of gamma irradiation on the activity of Streptomyces incanus BK128 towards PHB production was carried out. The strain was exposed to increasing doses of gamma radiation ( 0.25 to 3.5 kGy). The cell concentration, PHB concentration and PHB content were investigated. The data in Fig. (5) shows that low doses of gamma radiation from 0.25 to $2 \mathrm{kGy}$ may stimulate both growth and PHB production. The highest cell concentration and PHB concentration 5.54 and $3.39 \mathrm{gl}^{-1}$ were achieved at the dose $1.5 \mathrm{kGy}$, respectively with PHB content of $61.2 \%$. However, a decrease in the cell concentration accompanied by low PHB production was achieved by the increasing doses of gamma radiation ( $>2 \mathrm{kGy})$. Several studies recorded that low doses of gamma radiation may stimulate the microbial growth and metabolic activities [20-21]. Meanwhile, high doses of gamma radiation were proved to be inhibitory for both growth and enzymatic activities of microorganisms. The exposure of cells to ionizing radiation sets off a chain of reactions giving rise to chemical changes followed by metabolic or physiological changes. The irradiation presents an additional stress to the cells which tends to disturb their organization [22]. Irradiation effects have been shown to occur with proteins, enzymes, nucleic acid, lipids and carbohydrates, all which may have marked effects on the cell.

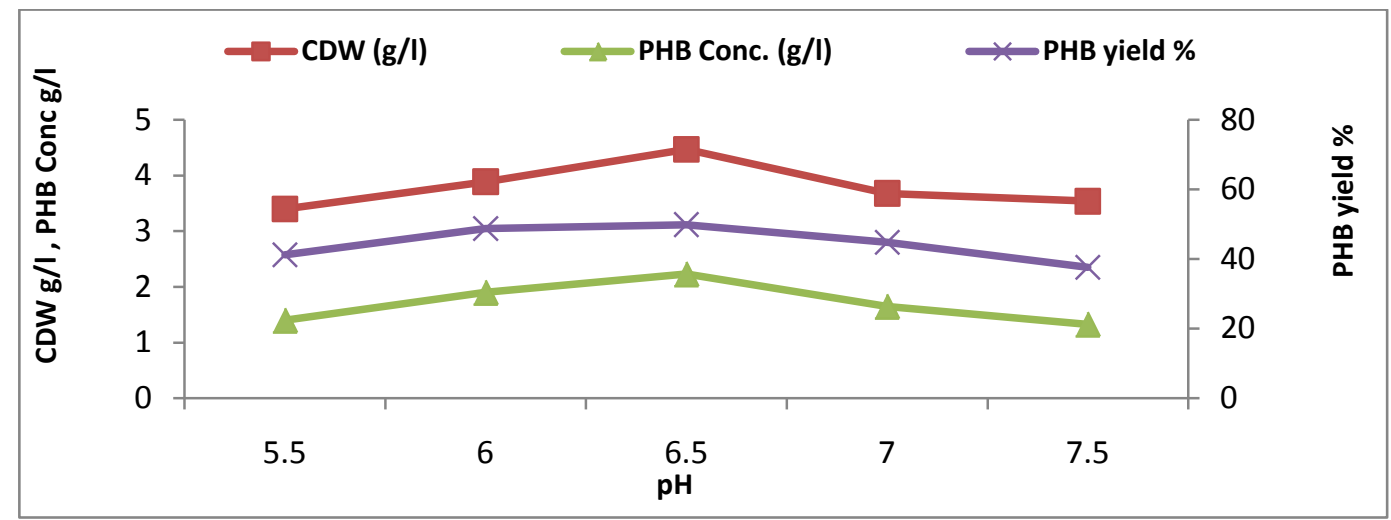

Fig. (1): Effect of pH on growth and PHB production from Streptomyces incanus BK128. 


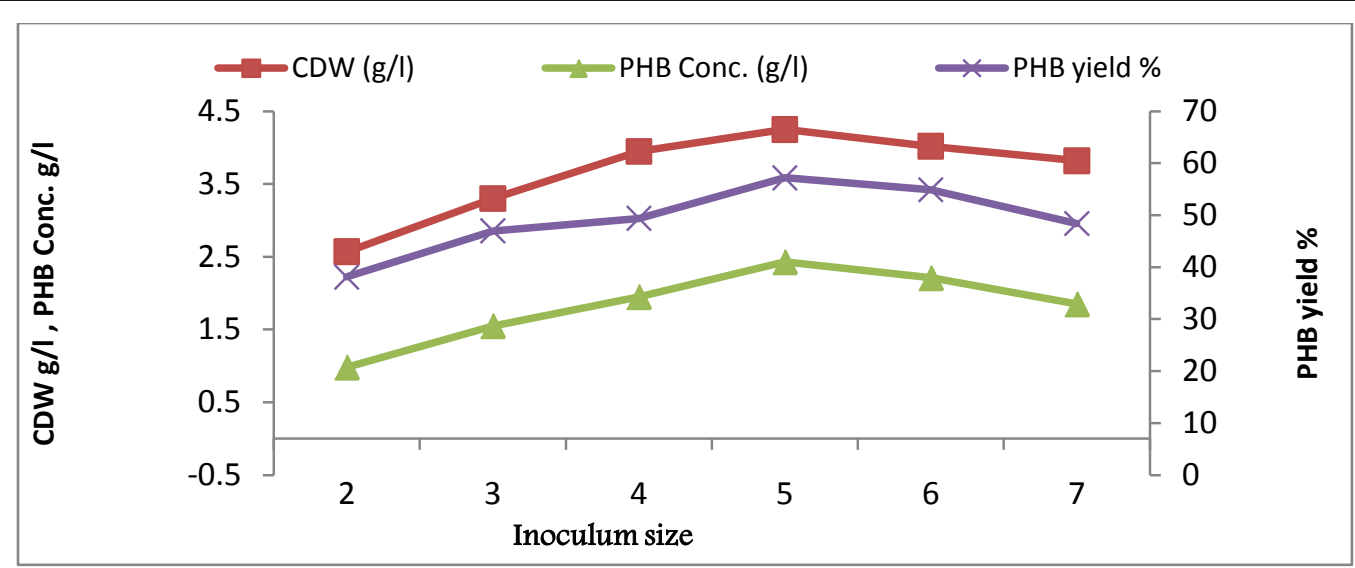

Fig. (2): Effect of inoculum size on growth and PHB production from Streptomyces incanus BK128.

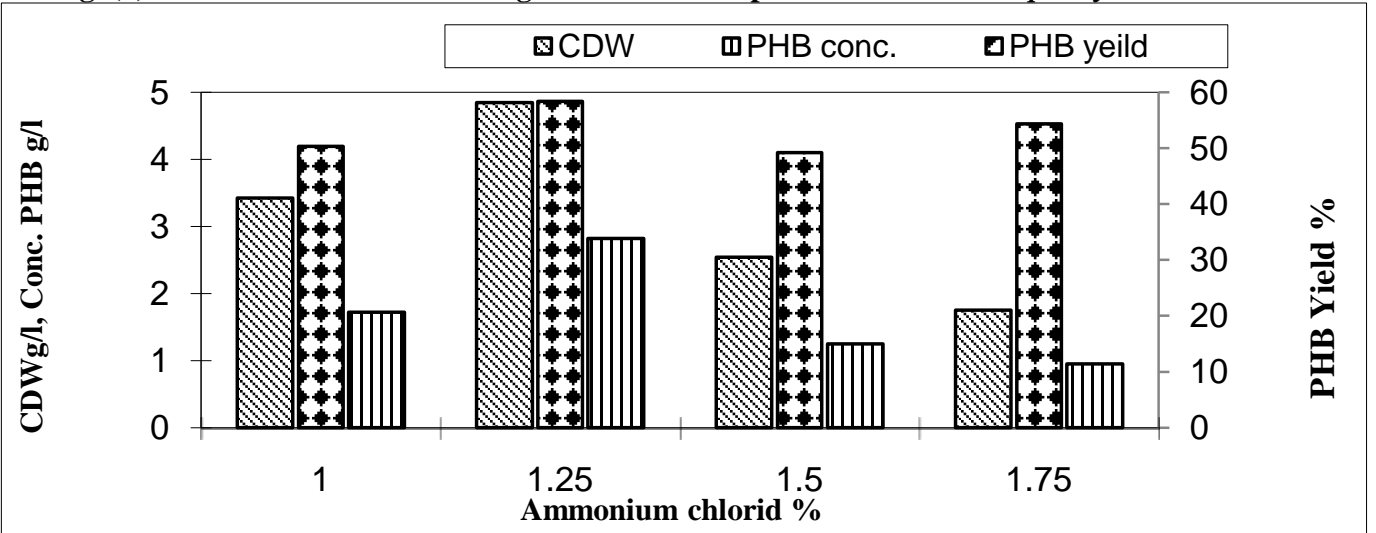

Fig. (3): Effect of $\mathrm{NH}_{4} \mathrm{CL}$ concentrations on growth and PHB production from Streptomyces incanus BK128.

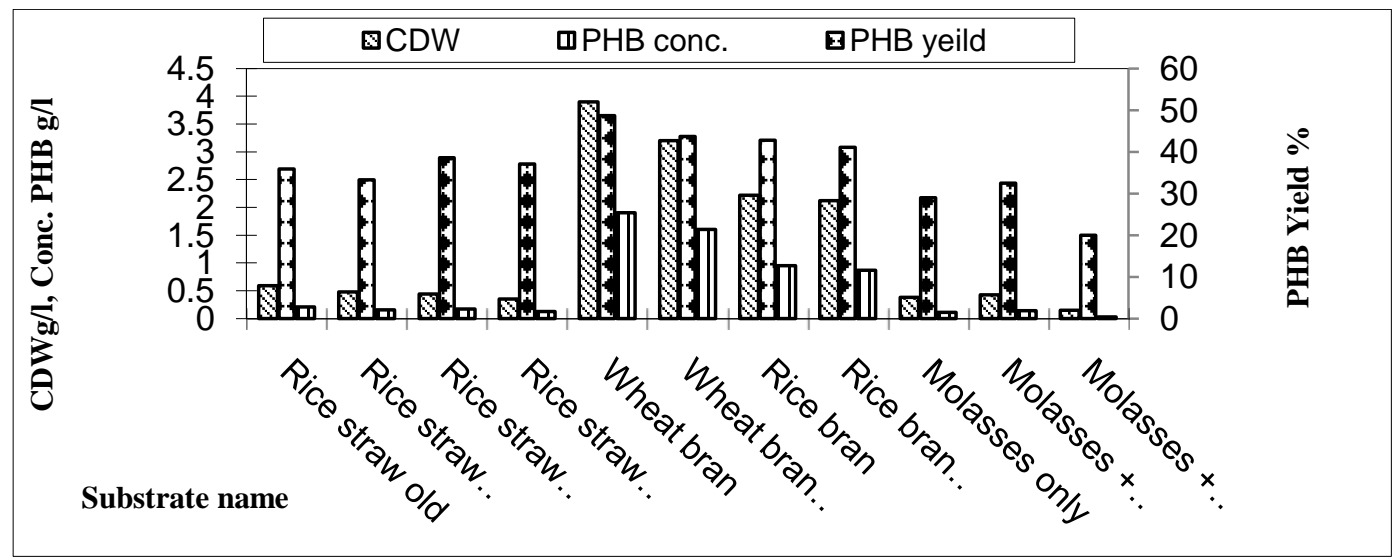

Fig. (4): Effect of different substrates on growth and PHB production from Streptomyces incanus BK128 


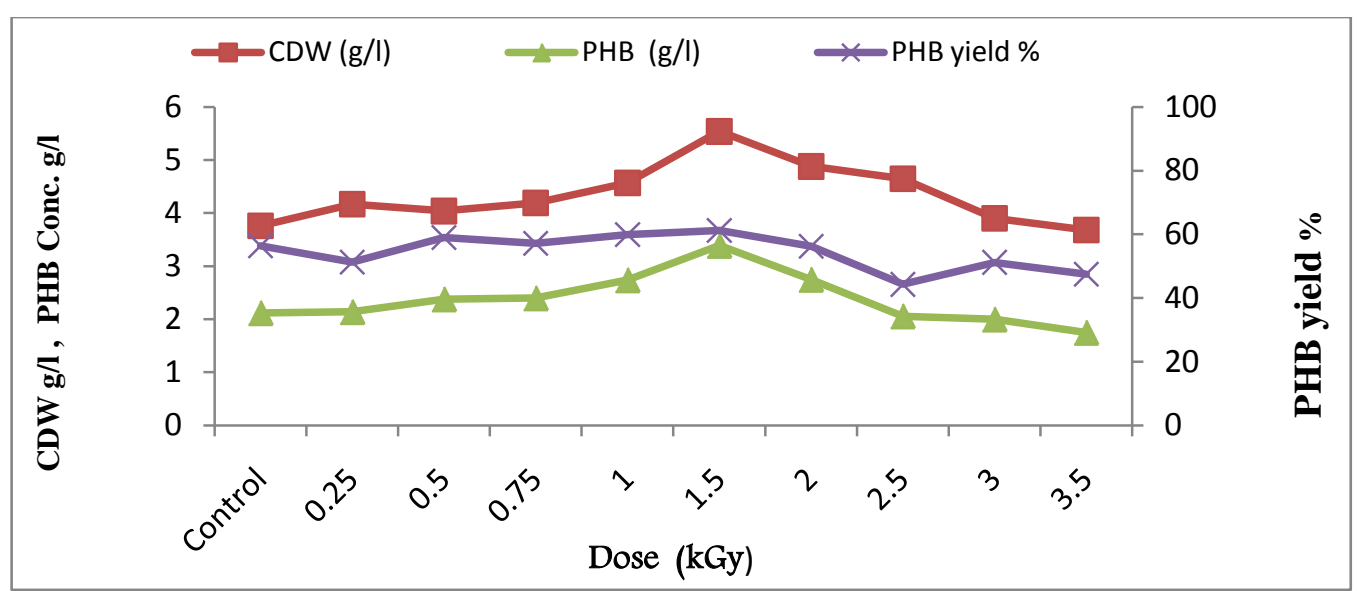

Fig. (5): Effect of gamma irradiation on the activity of Streptomyces incanus BK128 towards PHB production

\section{Conclusion}

This study proved that wheat bran can be used as an alternative to starch nitrate medium for PHB production. Optimization of flask culture conditions indicated that the maximum PHB production by Streptomyces incanus BK128 could be achieved on $100 \mathrm{ml}$ of starch nitrate medium with initial pH 6.5 at $30{ }^{\circ} \mathrm{C}$ for 5 days, $150 \mathrm{rpm}$ rotary shaker, and $5 \mathrm{ml}$ inoculum's size. These conditions resulted in 4.84 and $2.82 \mathrm{gl}^{-1}$ of cell dry weight and PHB dry weight, respectively with $56.9 \%$ of PHB content. However, both growth and PHB production were found to be stimulated by the exposure of Streptomyces incanus BK128 to low doses of gamma radiation (0.25-2 kGy). The highest cell concentration (5.54 $\left.\mathrm{gl}^{-1}\right)$ and PHB accumulation $\left(3.39 \mathrm{gl}^{-1}\right)$ was achieved at the dose 1.5 kGy with PHB content 61.2\%.

\section{References}

1. Wang, Y.; Yin, J.; Chen, G.Q. 2014. Polyhydroxyalkanoates, challenges and opportunities. Curr. Opin. Biotech. 30:59-65.

2. Palaniyandi, S.A.; Yang, S.H.; Suh, J.W 2013. Agricultural residues for production of cellulase from Sporotrichum thermophile LAR5 and its application for saccharification of rice straw. J. Basic. Microbiol.15:15-21.

3. Tripathi, A.D.; Srivastava, S.K.; Singh, R.P. 2013. Statistical optimization of physical process variables for bio-plastic (PHB) production by Alcaligenes sp. Biomass. Bioenergy. 55: 243-250.

4. Adamu A.A; Ibrahim, N.; John, O.; Matilda, A.O. 2017. Improvement of the production of microorganism strains by Using of mutagens. Folia Microbiol. 5: 102- 108.
5. Bibers, H. and Kalnis, M. 1999. Control of biopolymer poly- $\beta$-hydroxybutyrate characteristics by $\gamma$-irradiation, Mechanics of composite materials, Vol. 35, pp.169-178.

6. Kieser, T.; Bibb, M.J.; Buttner, M.J.; Chater, K.F.; Hopwood, D.A. 2000. Practical Streptomyces genetics. The John Innes Foundation, Norwich, U.K.

7. Shah, K.R. 2014. Optimization and production of Polyhydroxybutyrate (PHB) by Bacillus subtilis G1S1from soil. Int. J. Curr. Microbiol. App. Sci. 3:377-387.

8. Wolfender, J.L.; Nuzillard, J. Vander-Hoft, J.J.J.; Renault, J.H. 2018 . Production of PHB and $\mathrm{P}$ (3HB-co-3HV) biopolymers by Bacillus megaterium strain OU303A isolated from municipal sewage sludge. World. J. of Microbiol. Biotechnol. 25(3):391/397.

9. Sindhu, R.; Silviya, N.; Binod, P.; Pandey, A. 2013. Pentose-rich hydrolysate from acid pretreated rice straw as a carbon source for the production of poly-3-hydroxybutyrate. Biochem. Eng. J. 78:67- 72.

10. Shivakumar, S. 2012. Polyhydroxybutyrate (PHB) production using agro-industrial residue as substrate by Bacillus thuringiensis IAM 12077. Int. J. Chem. Tech. Res. 4:1158-1162.

11. Belal, E.B. 2013. Production of poly- $\beta$ hydroxybutyric acid (PHB) by Rhizobium elti and Pseudomonas stutzeri. Curr. Res. J. Biol. Sci. 5: 273-284.

12. Law, J.H. and Slepecky, R.A. 1961. Assay of poly- $\beta$-hydroxybutyric acid. J. Bacteriol., 82: 33- 36.

13. Nehra, K.; Dahiya, N.; Lathwal, P. and Singh, M. 2015. Influence of nutritional and environmental parameters on PHB production by bacteria isolated from 
contaminated soils. Int. J. Pharma Biosc., 6(1) (B): 676-691.

14. Shaaban, M.T.; Attia, M.; Turky, A.S.; Mowafy, E.I. 2012. Production of some biopolymers by some selective Egyptian soil bacterial isolates. J. Appl. Sci. Res. 8:94-105.

15. El-sayed, N.S.; Aboshanab, K.M.; Aboulwafa, M.M.; Hassouna, N.A. 2013. Optimization of bioplastic (poly- $\beta$-hydroxybutyrate) production by a promising Azomonas macrocytogenes bacterial isolates P173. Afr. J. Microbiol .Res. 7:5025-5035.

16. Thakur, P.S.; Borah, B.; Baruah, S.D.; Nigam, J.N. 2017. Synthesis of polyhydroxyalkanoate from hydrolyzed linseed oil. J. Environ. Poly. Degrade. 5: 153-158.

17. Van-Thuoc, D.; Huu-Phong, T.; Thi-Binh, N.; Thi-Tho, N.; Minh-Lam, D.; Quillaguama'n, J. 2012. Utilization of agricultural residues for poly (3-hydroxybutyrate) production by Halomonas boliviensis LC1. J. Appl. Microbiol. 104:420-428.

18. Lopez J.A.; Naranjo, J.M.; Higuita, J.C.; Cubitto, M.A.; Cardona, C.A.; Villar, M.A.
2012. Biosynthesis of PHB from a new isolated Bacillus megaterium strain: outlook on future developments with endospore forming bacteria. Biotechnol. Bioprocess. Eng. 17: 250258.

19. Bajaj, B.K.; and Sharma, P. 2011. An alkalithermotolerant extracellular protease from a newly isolated Streptomyces sp. DP2. New. Biotech .28:725-732.

20. IAEA, 1973. The effect of ionizing radiation on bacteria. In: Manual of radiation sterilization of medical and biological materials technical reports. Series No. 149.

21. Glusti, A.M.; Raimodi, M.; Ravagnan, G.; Sapora, O. and Parasass, T. 1988. Human cell membrane oxidative damage induced by single and functional doses of ionizing radiation. Fluorescence spectroscopy study. J. Rad. Biol. 74: 595-605.

22. Kim, J.I.; and Cox, M.M. 2002. The RecA proteins of Deinococcus radiodurans and Escherichia coli promote DNA strand exchange via inverse pathways. Proc. Natl. Acad. Sci. U. S. A. 99: 7917-7921. 